\title{
Spin Hanle effect in mesoscopic superconductors
}

\author{
M. Silaev, ${ }^{1,2}$ P. Virtanen, ${ }^{1}$ T. T. Heikkilä, ${ }^{3}$ and F. S. Bergeret ${ }^{4,5,6}$ \\ ${ }^{1}$ O.V. Lounasmaa Laboratory, P.O. Box 15100, FI-00076 Aalto University, Finland \\ ${ }^{2}$ Department of Theoretical Physics, The Royal Institute of Technology, Stockholm SE-10691, Sweden \\ ${ }^{3}$ Department of Physics and Nanoscience Center, University of Jyväskylä, P.O. Box 35 (YFL), FI-40014 University of Jyväskylä, Finland \\ ${ }^{4}$ Centro de Física de Materiales (CFM-MPC), Centro Mixto CSIC-UPV/EHU, Manuel de Lardizabal 5, E-20018 San Sebastián, Spain \\ ${ }^{5}$ Donostia International Physics Center (DIPC), Manuel de Lardizabal 5, E-20018 San Sebastián, Spain \\ ${ }^{6}$ Institut für Physik, Carl von Ossietzky Universität, D-26111 Oldenburg, Germany \\ (Received 7 August 2014; revised manuscript received 21 December 2014; published 14 January 2015)
}

\begin{abstract}
We present a theoretical study of spin transport in a superconducting mesoscopic spin valve under the action of a magnetic field misaligned with respect to the injected spin. We demonstrate that superconductivity can either strongly enhance or suppress the coherent spin rotation, depending on the type of spin relaxation mechanism being dominated either by spin-orbit coupling or spin-flip scattering at impurities. We also predict a subgap contribution to the nonlocal conductance in multiterminal superconducting hybrid structures which completely eliminates the effect of spin rotation at sufficiently low temperatures.
\end{abstract}

DOI: 10.1103/PhysRevB.91.024506

PACS number(s): 74.25.F-, 72.25.Ba, 72.25.Hg, 72.25.Rb

\section{INTRODUCTION}

Effective control over spin-polarized transport is a cornerstone for many spintronics applications [1]. One way of implementing spin manipulation and control is to exploit the Hanle effect, i.e., the coherent rotation of a spin in an external magnetic field [2]. Such rotation has been experimentally demonstrated in semiconducting nanostructures [3,4], graphene [5], and normal metals [6-9]. In the latter case, however, the strong spin-relaxation mechanisms impose a requirement of much larger magnetic fields to rotate the spin of rapidly moving electrons within a distance comparable to the spin coherence length [10].

One possible alternative to tune the spin rotation in metals is to use the intriguing spin transport properties of superconductors [11-13], which have recently stimulated broad interest and rapid progress in the emergent field of superconducting spintronics [14-16]. In contrast to the normal metals, the spin in superconductors is transported by the Bogoliubov quasiparticles, which move at the group velocity $v_{g} \sim v_{F} \sqrt{(\varepsilon / \Delta)^{2}-1}$ that tends to zero near the gap edge $\varepsilon=$ $\Delta$. Hence while traveling a fixed distance $L$ they are exposed to the spin-rotating field for a longer time, which results in an enhanced spin precession. This also implies an increase of the spin relaxation due to the exchange interaction with magnetic impurities [17-19]. However, besides generating the spin rotation and relaxation, the magnetic field induces a Zeeman splitting of quasiparticle states which can lead to the separation of spin and charge degrees of freedom [20] and a drastic suppression of spin relaxation $[12,13,21]$. Thus, in principle the magnetic field can either enhance the spin relaxation due to the Hanle mechanism or suppress it by the polarization of the quasiparticles. Although some works have been devoted to the theory of spin relaxation in superconductors [17-20], none of them have addressed the problem of noncollinear spin-splitting fields, essential to understand the Hanle effect in superconductors.

In this paper we address this problem and present a full study of spin transport in a typical nonlocal measurement setup. We extend the existing spin transport theory in diffusive superconductors $[17,18]$ by taking into account noncollinear configurations of spin injector and detector electrodes and an external magnetic field in an arbitrary direction. We show that the nonlocal magnetoresistance depends crucially on the spin relaxation mechanism in the superconductor. If the latter is mainly due to an extrinsic spin-orbit coupling, the nonlocal spin signal in the superconducting state is suppressed by smaller fields as compared to the normal case, and the period of characteristic oscillations of the Hanle curve becomes smaller. In contrast, if the main source of spin relaxation is due to magnetic impurities (spin-flip scattering), the decay length of the nonlocal spin signal with the applied field is longer in the superconducting state but the oscillations becomes less pronounced for $T<T_{c}$. Our theory also predicts that the injection of spins noncollinear with the external field can generate in the superconductor a subgap pure spin imbalance that provides an additional contribution to the subgap nonlocal conductance in multiterminal superconducting hybrid structures [22-24].

The structure of this paper is as follows. In Sec. II we describe a model of the nonlocal resistance measurements and the general Usadel theory in spin-polarized diffusive superconductors. In Sec. III we derive the equations describing spin injection, rotation, and decay of spin coherence in diffusive superconductors under the action of a transverse magnetic field. In Sec. IV we discuss in detail the Hanle effect and give the conclusions in Sec. V.

\section{MODEL}

\section{A. Nonlocal spin valve}

We consider the nonlocal spin valve shown in Fig. 1. A spin-polarized current is injected in the superconducting wire from a ferromagnetic electrode with magnetization $\boldsymbol{P}_{I}$. The detector is also a ferromagnet with a polarization $\boldsymbol{P}_{D}$ located at a distance $L_{D}$ from the injector. Both the injector and the detector are coupled to the wire via tunnel contacts. A magnetic field $\boldsymbol{B}$ is applied in $z$ direction. The current $I_{D}$ at the detector 


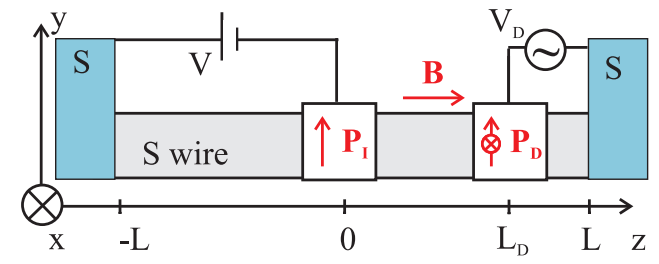

FIG. 1. (Color online) Schematic view of a nonlocal conductance measurement setup with noncollinear polarization of ferromagnetic contacts and magnetic field. We choose the injector polarization $\boldsymbol{P}_{I} \|$ $\boldsymbol{y}$, the magnetic field $\boldsymbol{B} \| \boldsymbol{z}$, while the detector polarization $\boldsymbol{P}_{D}$ has an arbitrary direction.

is given by (below, we use $\hbar=e=k_{B}=1$ )

$$
R_{D} I_{D}=\mu_{0}+\boldsymbol{\mu} \cdot \boldsymbol{P}_{D}
$$

where $R_{D}$ is the detector interface resistance in the normal state, $\mu_{0}$ is the effective charge imbalance of quasiparticles in the superconductor, and the last term describes the spindependent contribution to the current which is proportional to the local spin accumulation $\boldsymbol{\mu}$. Expression (1) follows directly from the boundary conditions at the spin-polarized ferromagnet-superconductor interface, as we show in Sec. II C.

In a nonlocal measurement scheme, the spin accumulation is tested by measuring the voltage at the detector where no charge current flows $[12,19]$, i.e., one sets in Eq. (1) $I_{D}=0$. The spin-dependent voltage $V_{S}$ is defined as the difference of voltages measured in the parallel and antiparallel configurations between the injector and the detector. The nonlocal spin signal is determined by the ratio $R_{S}=V_{S} / I_{\mathrm{inj}}$, where $I_{\text {inj }}=V \chi / R_{I}$ is the injected current, $R_{I}$ is the injector interface resistance, and $\chi=\int_{0}^{\infty} d \varepsilon N_{+} \frac{\partial n_{0}}{\partial \varepsilon}$ is the "Yosida function"[19]. Here $N_{+}$is the density of states (DOS) in the superconductor near the ferromagnetic electrode and $n_{0}(\epsilon)$ is the Fermi distribution function. As usual, we consider only the linear response limit $|V| \ll T$. The divergence of the spin signal in the low-temperature limit [25] is cut off by proximity-induced subgap contributions to $N_{+}$. The corresponding nonlocal resistance is

$$
R_{S}=2 R_{I}\left(V \chi^{2}\right)^{-1}\left(\boldsymbol{\mu} \cdot \boldsymbol{P}_{\boldsymbol{D}}\right),
$$

as obtained from Eq. (1). Below we calculate in detail the spin accumulation $\mu$ in a superconductor with Zeeman splitting and discuss the resulting behavior of $R_{S}$.

\section{B. Nonequilibrium Green's functions and the Usadel equation}

The spin accumulation $\boldsymbol{\mu}$ in Eqs. (1) and (2) can be written in terms of the Keldysh quasiclassical Green's function (GF) as [26]

$$
\boldsymbol{\mu}=\int_{0}^{\infty} \boldsymbol{m}(\varepsilon) d \varepsilon,
$$

where $\boldsymbol{m}(\varepsilon)=\operatorname{Tr}\left(\tau_{3} S g^{K}\right) / 8, \tau_{3}$ is the third Pauli matrix in Nambu space, $\boldsymbol{S}=\left(\sigma_{1}, \sigma_{2}, \sigma_{3}\right)$ is the vector of Pauli matrices in spin space, and $g^{K}$ is the $(2 \times 2$ matrix $)$ Keldysh component of the quasiclassical GF matrix

$$
\check{g}=\left(\begin{array}{cc}
g^{R} & g^{K} \\
0 & g^{A}
\end{array}\right),
$$

and $g^{R(A)}$ is the retarded (advanced) GF. In a diffusive superconducting wire the matrix $\breve{g}$ obeys the Usadel equation [27]

$$
\frac{D}{2} \nabla \cdot(\check{g} \nabla \check{g})+\left[\check{\Lambda}-\check{\Sigma}_{\text {so }}-\check{\Sigma}_{\text {sf }}, \check{g}\right]=0 .
$$

Here $D$ is the diffusion constant, $\breve{\Lambda}=i \varepsilon \tau_{3}-i(\boldsymbol{h} \cdot \boldsymbol{S}) \tau_{3}-\check{\Delta}$, $\varepsilon$ is the energy, $\breve{\Delta}=\Delta \tau_{1}$ the spatially homogeneous order parameter in the wire, $\boldsymbol{h}=\mu_{B} \boldsymbol{B}$ the Zeeman field, $\mu_{B}$ the Bohr magneton. The last two terms in Eq. (5), $\check{\Sigma}_{\mathrm{so}}=\tau_{\mathrm{so}}^{-1}(\boldsymbol{S} \cdot \check{g} \boldsymbol{S})$ and $\check{\Sigma}_{\text {sf }}=\tau_{\text {sf }}^{-1}\left(\boldsymbol{S} \cdot \tau_{3} \check{g} \tau_{3} S\right)$, describe spin relaxation due to spin-orbit scattering and exchange interaction with magnetic impurities, characterized by relaxation times $\tau_{\mathrm{so}}$ and $\tau_{\mathrm{sf}}$, respectively. The commutator is defined as $[A, B]=(A B-$ $B A) / 2$. Equation (5) is complemented by the normalization condition $\check{g}^{2}=1$ that allows writing the Keldysh component as $g^{K}=g^{R} \hat{f}-\hat{f} g^{A}$, where $\hat{f}$ is the distribution function with a general spin structure

$$
\hat{f}=f_{L}+f_{T} \tau_{3}+\left(\boldsymbol{\sigma} \cdot \boldsymbol{f}_{T}\right)+\left(\boldsymbol{\sigma} \cdot \boldsymbol{f}_{L}\right) \tau_{3} .
$$

Here the $L$-labeled functions denote the (spin) energy degrees of freedom and are always antisymmetric with respect to the Fermi level $\varepsilon=0$ in the superconductor. The $T$-labeled functions are symmetric and describe the charge or spin imbalance. This is a generalization of the Schmid-Schön theory to the case of noncollinear spin transport $[17,18,28]$.

We assume that the transparencies of the detector and injector interfaces are small, so that up to leading order the spectral (retarded and advanced) GFs obtain their bulk values in the presence of a Zeeman splitting field. In the present case $\boldsymbol{h}=h \boldsymbol{z}$, they read

$$
g^{R}=\tau_{1}\left(g_{01}+g_{31} \sigma_{3}\right)+\tau_{3}\left(g_{03}+g_{33} \sigma_{3}\right),
$$

and $g^{A}=-\tau_{3} g^{R \dagger} \tau_{3}$. While the diagonal terms in Nambu space $\left(\tau_{3}\right)$ correspond to the normal GFs, $g_{01}, g_{31}$ are the singlet and zero-spin-triplet anomalous components which describe the superconducting condensate [27]. The coefficients in Eq. (7) are determined by solving the nonlinear equation

$$
\left[\Lambda^{R}-\Sigma_{\mathrm{sf}}^{R}-\Sigma_{\mathrm{so}}^{R}, g^{R}\right]=0,
$$

where the spin-dependent scattering self-energies reduce the (self-consistent) spectral gap to $\Delta_{g}<\Delta-h$ and smear the gap edge singularities [29], as shown in Fig. 2. Apart from a temperature interval in the vicinity of $T_{c}$, the applied fields are much smaller than the paramagnetic Chandrasekhar-Clogston limit [30] $|h| \ll \Delta / \sqrt{2}$.

\section{Currents and boundary conditions}

In terms of the GF [Eq. (4)], the bulk currents are given by

$$
\boldsymbol{j}_{\mathrm{ki}}=\frac{\sigma_{N}}{8} \int_{0}^{\infty} d \varepsilon \operatorname{Tr} \tau_{k} \sigma_{i}(\check{g} \nabla \check{g})^{K},
$$

where $\sigma_{N}$ is the normal-state conductivity, $k=0,3, i=$ $0,1,2,3$ and the sets of indices $(k=3 ; i=0),(k=0 ; i=0)$, $(k=0 ; i=1,2,3)$, and $(k=3 ; i=1,2,3)$ correspond to the charge, energy, spin, and spin energy currents, respectively.

The GF has to be determined from the Usadel equation (5), which is completed by boundary conditions (BCs) at the spin-polarized injector interface $z=0$. We use here the BCs 
(a) $\beta=0.5$
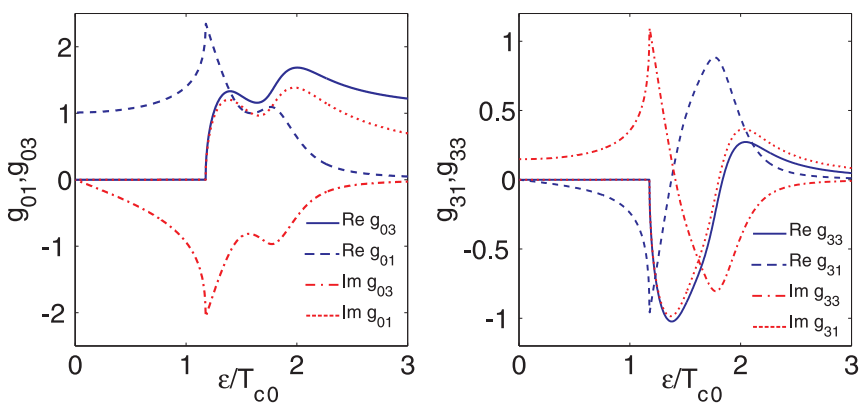

(b) $\beta=-0.9$
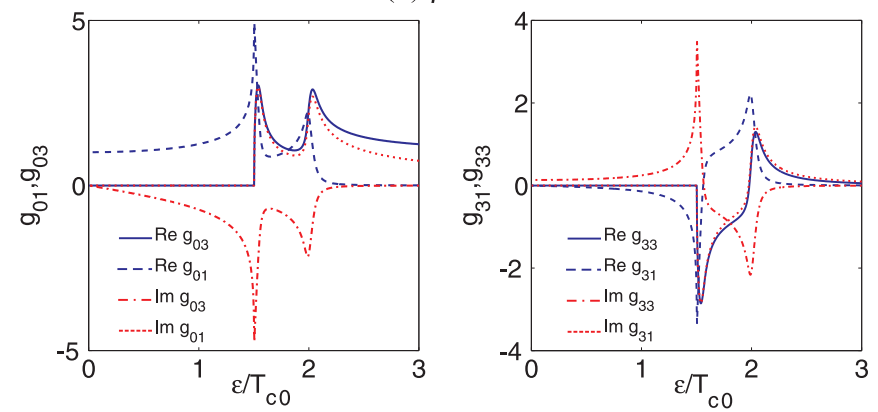

FIG. 2. (Color online) Components of the spectral GF $g^{R}$ for the different relative strengths of spin-flip and spin-orbital scattering (a) $\beta=0.5$ (dominating spin-flip scattering) and (b) $\beta=-0.9$ (dominating spin-orbital scattering). Left and right panels show spinsinglet and spin-triplet GF components, respectively. The component $N_{+}=\operatorname{Re} g_{03}$ is the total DOS in spin subbands. The parameters are $T=0.25 T_{c 0}, \tau_{\mathrm{sn}}=5 / T_{c 0}$, and $h=1.25 / \tau_{\mathrm{sn}}$.

of Ref. [31] that generalizes the Kupriyanov-Lukichev [32] condition to the case of a spin-dependent barrier transmission. These BCs are obtained by matching the bulk currents in the superconductor with the tunneling currents through the interface.

The currents through a ferromagnetic barrier characterized by an effective polarization $\boldsymbol{P}=P \boldsymbol{z}$ are given by [31]

$$
\boldsymbol{j}_{\mathrm{ki}}^{t}=\frac{1}{8 R_{\square}} \int_{0}^{\infty} d \varepsilon \operatorname{Tr} \tau_{k} \sigma_{i}\left[\hat{\Gamma} \check{g}_{N} \hat{\Gamma}^{+}, \check{g}\right]^{K} .
$$

Here $R_{\square}$ is the barrier resistance per unit area, and $\check{g}_{N}$ is the matrix GF of the normal electrode. The components of $\check{g}_{N}$ are given by $g_{N}^{R(A)}= \pm \sigma_{0} \tau_{3}$ and $g_{N}^{K}=2\left(n_{-}+\tau_{3} n_{+}\right)$, where $n_{ \pm}=$ $\left[n_{0}(\varepsilon+V) \pm n_{0}(\varepsilon-V)\right] / 2$ is the voltage-biased distribution function in the normal metal electrode, $n_{0}(\varepsilon)=\tanh (\varepsilon / 2 T)$. The spin-filtering tunneling matrix $\hat{\Gamma}=t \tau_{3}+u \sigma_{3}$ is defined through the normalized transparencies $t^{2}+u^{2}=1$ which satisfy the condition $u t=P / 2$.

Throughout this work we assume that the applied field is in $z$ direction, while the magnetization of the injector and detector may point in another direction. Noncollinear polarization of the barrier, e.g., rotated by the angle $\alpha$ in the $y z$ plane, $\boldsymbol{P}=$ $P(\cos \alpha z+\sin \alpha y)$, corresponds to spin rotation of the GF in the superconductor around the $x$ axis,

$$
\begin{gathered}
\check{g}=\hat{R}^{+} \check{g}_{\text {new }} \hat{R}, \\
\hat{R}=e^{i \alpha \sigma_{1} / 2},
\end{gathered}
$$

which modifies the transparency matrix as $\hat{\tilde{\Gamma}}=\hat{R} \hat{\Gamma}$. Equation (1) for the current measured by the detector electrode follows directly from Eqs. (10) and (11) by choosing $\boldsymbol{P}=\boldsymbol{P}_{D}$ and $R_{D}=R_{\square} / A_{D}$, where $A_{D}$ is the effective area of the detector.

The BCs at the injector electrode are obtained from the conservation of the spectral current density $\mathbf{j}_{\mathrm{ki}}=\mathbf{j}_{\mathrm{ki}}^{t}$ and have the form

$$
\check{g} \nabla \check{g}=\kappa_{I}\left[\tilde{g}_{N}, \check{g}\right],
$$

where $\kappa_{I}=1 /\left(R_{I \square} \sigma_{N}\right)$ is the injector transparency and $\tilde{g}_{N}=\hat{\tilde{\Gamma}} \check{g}_{N} \hat{\tilde{\Gamma}}^{+}$. Let us consider the matrix BC Eq. (13) in components. The $R, A$ elements yield

$$
\begin{aligned}
& g^{R} \nabla g^{R}=\kappa_{I}\left[\tilde{g}_{N}^{R}, g^{R}\right], \\
& g^{A} \nabla g^{A}=\kappa_{I}\left[\tilde{g}_{N}^{A}, g^{A}\right],
\end{aligned}
$$

and the Keldysh component can be written as follows:

$$
\begin{aligned}
& \nabla f-g^{R} \nabla f g^{A}+g^{R} \nabla g^{R} f-f g^{A} \nabla g^{A} \\
& \quad=\kappa_{I}\left(\tilde{g}_{N}^{R} g^{K}+\tilde{g}_{N}^{K} g^{A}-g^{R} \tilde{g}_{N}^{K}-g^{K} \tilde{g}_{N}^{A}\right) / 2 .
\end{aligned}
$$

With the help of Eqs. (14) and (15), the above expression can be simplified:

$$
\begin{aligned}
\nabla f-g^{R} \nabla f g^{A}= & \kappa_{I}\left(\tilde{g}_{N}^{R} \tilde{f} g^{A}+g^{R} \tilde{f} \tilde{g}_{N}^{A}\right. \\
& \left.-g^{R} \tilde{g}_{N}^{R} \tilde{f}-\tilde{f} \tilde{g}_{N}^{A} g^{A}\right) / 2,
\end{aligned}
$$

where $\tilde{f}=f_{N}-f$ is the difference between distribution functions in the superconducting wire (6) and in the normal metal electrode $f_{N}=n_{+}+n_{-} \tau_{3}$.

In the next sections we discuss the relaxation and precession of the spin in a diffusive superconductor by solving the boundary problem described by Eqs. (5), (14), (15), and (17).

\section{SPIN PRECESSION AND RELAXATION IN A DIFFUSIVE SUPERCONDUCTOR}

Although the coherent precession of spin has been studied extensively in a number of semiconducting and normal metal systems [3-9], a theory for superconductors is still lacking. In this section we derive, from the general expressions presented in the previous section, a compact set of kinetic equations and boundary conditions which describe the injection of a transverse spin polarization, its precession, and relaxation. In the next section we solve these equations and address the spin precession measurable in nonlocal spin valves (see Fig. 1), known as the Hanle effect.

For simplicity, we fix the directions of the injecting electrode polarization to $\boldsymbol{P}_{I}=P_{I} \boldsymbol{y}$ and the Zeeman field $\boldsymbol{h}=h \boldsymbol{z}$, and allow the polarization of the detector to have an arbitrary direction. In this case $\mu_{z}=0$, and to evaluate the nonlocal electric signal (1) it is sufficient to consider only a transversal component of the spectral spin polarization $\mathbf{m}_{\perp}=\left(m_{x}, m_{y}, 0\right)$, which is given by

$$
\boldsymbol{m}_{\perp}=N_{+} \boldsymbol{f}_{T}+h^{-1} \operatorname{Im} g_{33}\left(\boldsymbol{f}_{T} \times \boldsymbol{h}\right),
$$

where $\boldsymbol{f}_{T}=\left(f_{T 1}, f_{T 2}, 0\right)$. The first term in Eq. (18) describes the quasiparticle contribution. It is proportional to the total DOS $N_{+}=\operatorname{Re} g_{03}$ modified by the Zeeman splitting and the 
spin-dependent scattering mechanisms (see Fig. 2, left panels). This contribution is only finite for energies above the spectral gap $\varepsilon>\Delta_{g}$. The second term in Eq. (18), being proportional to $\operatorname{Im} g_{33}$, is nonzero only if the superconducting spectrum is spin polarized. In contrast to the usual quasiparticle contribution, this term is not suppressed at low temperatures $T \ll T_{c}$ since $\operatorname{Im} g_{33}$ is nonzero at subgap energies [dash-dotted curve in Fig. 2(b)]. As we demonstrate below, this term leads to a finite subgap nonlocal conductance in the lowest order in transparency. This contribution involves only spin degrees of freedom, in contrast to previous works on the subgap charge transport [22-24].

Let us derive the equations for the transverse distribution function $\boldsymbol{f}_{T}$ from the general equation (5). These components are decoupled from the others and satisfy the kinetic equations

$$
\begin{aligned}
& \mathcal{D}_{T 1} \nabla^{2} f_{T 1}+\mathcal{D}_{T 2} \nabla^{2} f_{T 2}=X_{1} f_{T 1}+X_{2} f_{T 2}, \\
& \mathcal{D}_{T 1} \nabla^{2} f_{T 2}-\mathcal{D}_{T 2} \nabla^{2} f_{T 1}=X_{1} f_{T 2}-X_{2} f_{T 1},
\end{aligned}
$$

where the renormalized diffusion coefficients are given by

$$
\begin{gathered}
\mathcal{D}_{T 1}=1+\left|g_{03}\right|^{2}-\left|g_{01}\right|^{2}+\left|g_{31}\right|^{2}-\left|g_{33}\right|^{2}, \\
\mathcal{D}_{T 2}=2 \operatorname{Im}\left(g_{33} g_{03}^{*}-g_{31} g_{01}^{*}\right) .
\end{gathered}
$$

In Eqs. (19) and (20) we have defined $X_{1}=\left(S_{T 1}-H_{1}\right) / D$ and $X_{2}=\left(S_{T 2}+H_{2}\right) / D$, where

$$
\begin{gathered}
H_{1}=4 h \operatorname{Im} g_{33}, \quad H_{2}=4 h N_{+}, \\
S_{T 1}=2 \tau_{\mathrm{sn}}^{-1}\left[\left(\operatorname{Re} g_{03}\right)^{2}+\beta\left(\operatorname{Im} g_{01}\right)^{2}\right], \\
S_{T 2}=2 \tau_{\mathrm{sn}}^{-1}\left(\operatorname{Im} g_{33} \operatorname{Re} g_{03}-\beta \operatorname{Im} g_{01} \operatorname{Re} g_{31}\right) .
\end{gathered}
$$

\section{(a) $\beta=0.5$}
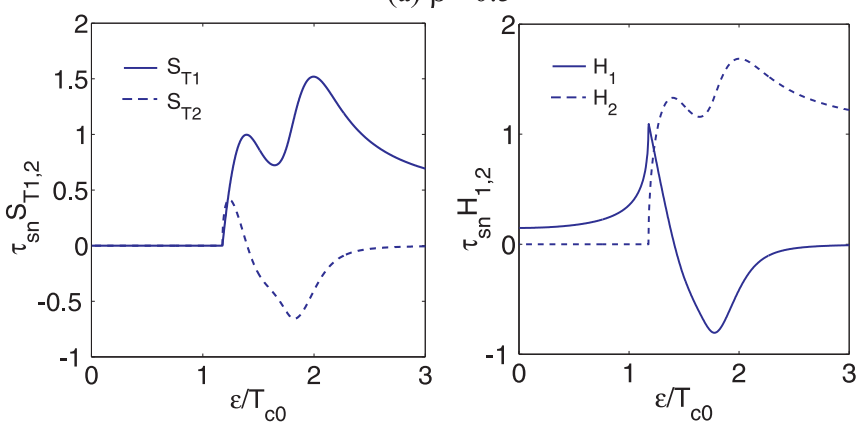

(b) $\beta=-0.9$
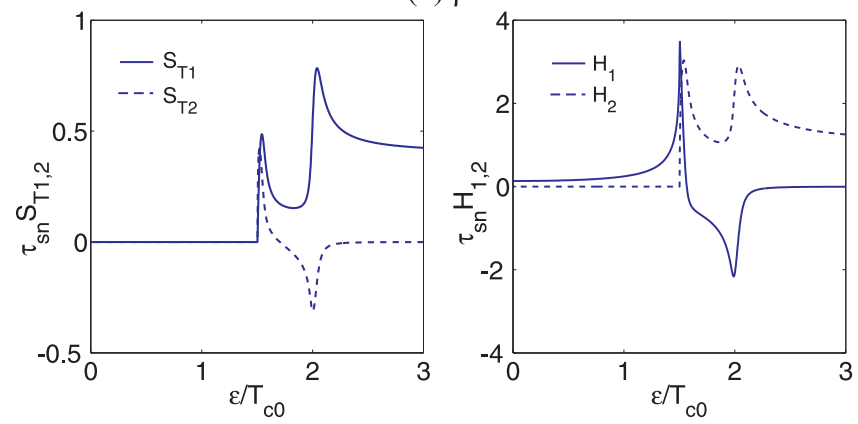

FIG. 3. (Color online) Energy dependencies of the spin relaxation terms $S_{1,2}$ (left panels) and Hanle terms $H_{1,2}$ (right panels) for $h=1.25 / \tau_{\mathrm{sn}}$, (a) $\beta=0.5$ and (b) $\beta=-0.9$.
Here the "Hanle" terms, Eq. (23), describe coherent spin rotation and relaxation due to the randomization of the spin precession phase under the action of the external magnetic field. Another source of spin relaxation is the elastic spinflip and spin-orbital scattering described by the terms of Eqs. (24) and (25). These terms are determined by two parameters which are the normal-state spin relaxation time $\tau_{\mathrm{sn}}=\tau_{\mathrm{sf}} \tau_{\mathrm{so}} / 8\left(\tau_{\mathrm{sf}}+\tau_{\mathrm{so}}\right)$ and the relative strength of spinorbital and spin-flip scattering $\beta=\left(\tau_{\mathrm{so}}-\tau_{\mathrm{sf}}\right) /\left(\tau_{\mathrm{so}}+\tau_{\mathrm{sf}}\right)$. For example, in $\mathrm{Al}$ wires used in the spin-transport experiments, the typical spin relaxation time is $\tau_{\mathrm{sn}} \approx 100 \mathrm{ps} \approx 5 / T_{c 0}$, where $T_{c 0} \approx 1.6 \mathrm{~K}$ is the bare critical temperature of the superconductor in the absence of the exchange field [19]. In $\mathrm{Al}, \beta=0.5$, indicating the dominating spin-flip relaxation mechanism [19], while for $\mathrm{Nb}$, one expects the spin orbit as the main source of scattering [21].

It is important for the discussions below to understand the energy dependencies of the spin relaxation, and Hanle terms, which are shown in Fig. 3 for spin-flip $\beta=0.5$ (a) and spinorbital $\beta=-0.9$ (b) dominated scattering. Correspondingly, we show in the left panels of Fig. 4 the dependencies of the diffusion coefficients $\mathcal{D}_{T 1,2}$.

In order to calculate the spin-dependent transport, we need the BCs at the injector interface $z=0$ which are determined from the general ones (18) and have the form

$$
\begin{aligned}
& \mathcal{D}_{T 1} \nabla f_{T 1}+\mathcal{D}_{T 2} \nabla f_{T 2} \\
& \quad=2 \kappa_{I}\left\{N_{+} f_{T 1}+\operatorname{Im} g_{33}\left[f_{T 2}+P_{I}\left(f_{T}-n_{-}\right)\right]\right\}, \\
& \mathcal{D}_{T 1} \nabla f_{T 2}-\mathcal{D}_{T 2} \nabla f_{T 1} \\
& \quad=2 \kappa_{I}\left\{N_{+}\left[P_{I}\left(f_{T}-n_{-}\right)+f_{T 2}\right]-\operatorname{Im} g_{33} f_{T 1}\right\} .
\end{aligned}
$$

(a) $\beta=0.5$
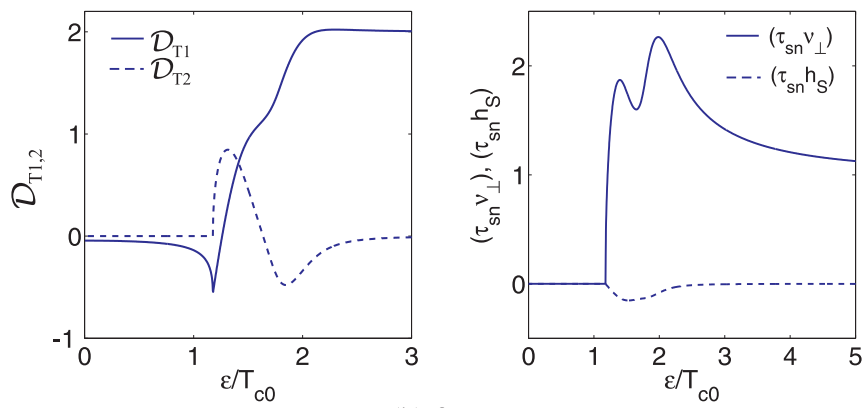

(b) $\beta=-0.9$
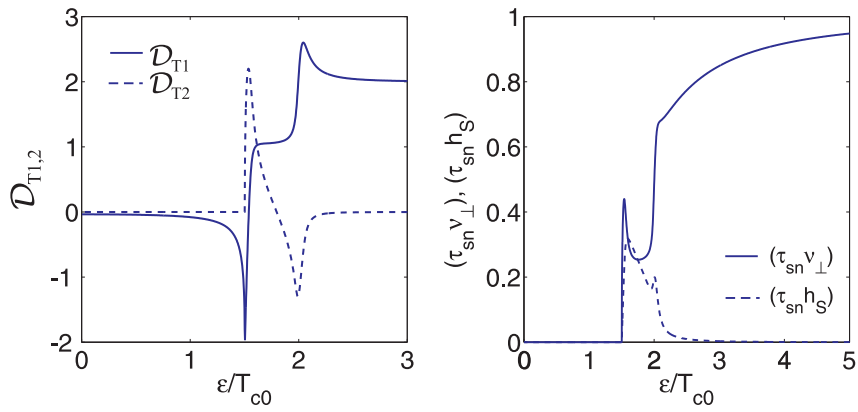

FIG. 4. (Color online) Energy dependencies of the diffusion coefficients $\mathcal{D}_{T 1,2}$ (left panels) and the characteristic frequencies of the spin diffusion equation (30) which are the spin coherence decay rate $v_{\perp}=1 / \tau_{\perp}$ and the precession frequency shift $h_{s}$. The plots are shown for $h=1.25 / \tau_{\mathrm{sn}}$, (a) $\beta=0.5$ and (b) $\beta=-0.9$. 
These BCs are valid for arbitrary values of transparency $\kappa_{I}$. They can be simplified in the spin injection limit $\kappa_{I} \xi \ll 1$, where $\xi=\sqrt{D / \Delta}$ is the superconducting coherence length. By omitting in the right-hand side of Eqs. (26) and (27) the terms which are of the second order in $\kappa_{I} \xi$ we get

$$
\begin{gathered}
\mathcal{D}_{T 1} \nabla f_{T 1}+\mathcal{D}_{T 2} \nabla f_{T 2}=-2 \kappa_{I} \operatorname{Im} g_{33} P_{I} n_{-}, \\
\mathcal{D}_{T 1} \nabla f_{T 2}-\mathcal{D}_{T 2} \nabla f_{T 1}=-2 \kappa_{I} N_{+} P_{I} n_{-} .
\end{gathered}
$$

At first glance, both the kinetic equations (19) and (20) and the BCs (26) and (27) have a rather complicated form. However, their physical meaning is quite clear, as we discuss in what follows. First, let us note that the kinetic equations (19) and (20) can be written in a more compact form similar to the Landau-Lifshitz-Gilbert equation for a damped gyromagnetic precession for the spectral density of spin polarization (18):

$$
\nabla \cdot \boldsymbol{j}_{s}=g_{S} \boldsymbol{m}_{\perp} \times\left(\boldsymbol{h}+\boldsymbol{h}_{s}\right)+\boldsymbol{m}_{\perp} / \tau_{\perp},
$$

where we have introduced an electronic spin $g$ factor $g_{S}=2$ and the transversal spin current density

$$
\boldsymbol{j}_{s}=\frac{D}{2}\left(\mathcal{D}_{T 1} \nabla \boldsymbol{f}_{T}-\mathcal{D}_{T 2} \hat{z} \times \nabla \boldsymbol{f}_{T}\right),
$$

where $\hat{z}=(0,0,1)$. In Eq. (30) the transverse spin relaxation time $\tau_{\perp}$ is given by

$$
\tau_{\perp}^{-1}=\frac{2 h\left(H_{2} S_{T 1}+H_{1} S_{T 2}\right)}{H_{1}^{2}+H_{2}^{2}} .
$$

The time $\tau_{\perp}$ determines the rate of spin coherence relaxation in the diffusive superconductor [33]. In contrast to the normal metal where it coincides with the usual spin relaxation time $\tau_{\perp}=\tau_{\mathrm{sn}}$, in the superconducting state $\tau_{\perp}$ has a pronounced energy dependence shown in the right panels of Fig. 4. The spin relaxation terms $S_{T 1, T 2}$ not only determine the decay of spin coherence but also introduce a shift of the precession frequency, which can be expressed as a renormalization of the Zeeman field [see the first term on the right-hand side of Eq. (30)]:

$$
\boldsymbol{h}_{s}=\boldsymbol{h} \frac{H_{2} S_{T 2}-H_{1} S_{T 1}}{H_{1}^{2}+H_{2}^{2}} .
$$

Also, this term is energy dependent, as shown in the right panels of Fig. 4.

The BCs (26) and (27) can be also written in a simple form using the definition of the spin current (31). We find

$$
\left.\boldsymbol{j}_{s}\right|_{z=0}=D \kappa_{I}\left(\left.\boldsymbol{m}_{\perp}\right|_{z=0}-\boldsymbol{m}_{N}\right),
$$

where $\boldsymbol{m}_{N}=\left(n_{-}-f_{T}\right)\left[N_{+} \boldsymbol{P}_{I}+h^{-1} \operatorname{Im} g_{33}\left(\boldsymbol{P}_{I} \times \boldsymbol{h}\right)\right]$ is the spin polarization acquired by nonequilibrium electrons which tunnel through the spin-filtering ferromagnetic barrier from the voltage-biased normal electrode to the superconductor. The above expression for $\boldsymbol{m}_{N}$ is given by Eq. (18) with $\boldsymbol{f}_{T}=$ $\boldsymbol{P}_{I}\left(n_{-}-f_{T}\right)$. The BCs in the spin injection limit $(28,29)$, are obtained by setting $\left.\boldsymbol{m}_{\perp}\right|_{z=0}=0$ and $f_{T}=0$ in the right-hand side of Eq. (34).

By combining Eqs. (18) and (34) one can easily verify that there is a finite subgap contribution to the spectral spin current originating from the second term in the right-hand side of Eq. (18). It is important to emphasize that this subgap spin imbalance appears in linear order in $\kappa_{I}$ and exists exclusively in the presence of a Zeeman field and noncollinear spin injection, $\boldsymbol{h} \nVdash \boldsymbol{P}_{I}$.

\section{HANLE EFFECT}

Let us now apply the general theory developed in the previous section to study the Hanle effect in the model of a nonlocal spin valve discussed in Sec. II. In this effectively one-dimensional (1D) system with a spatially homogeneous distribution of the order parameter, Eq. (30) can be solved analytically. The components of $\boldsymbol{f}_{T}$ have the form $f_{T 1}=$ $-\operatorname{Im}\left(A e^{-k_{T} z}\right)$ and $f_{T 2}=\operatorname{Re}\left(A e^{-k_{T} z}\right)$, where $A$ is an integration constant determined by the BCs, and

$$
k_{T}=\left[\frac{\left(S_{T 1}-H_{1}\right)-i\left(S_{T 2}+H_{2}\right)}{D\left(\mathcal{D}_{T 1}-i \mathcal{D}_{T 2}\right)}\right]^{1 / 2}
$$

is the inverse of the characteristic length with $\operatorname{Re} k_{T}>0$. Its real part determines the inverse spin relaxation length which is energy dependent. Its imaginary part describes the precession of the spin of quasiparticles with energy $\varepsilon$. As can be seen from Figs. 5(a) and 5(b), the precession and relaxation lengths depend on the nature of the spin-scattering mechanism. At intermediate temperatures below $T_{c}$, the main contribution to the spin-dependent $\boldsymbol{\mu}$ in Eq. (2) comes from energies close to the spectral gap $\Delta_{g}$. In the case of dominating spin-orbit scattering $(\beta<0)$, one clearly sees in Figs. 5(a) and 5(b) that while $\operatorname{Re} k_{T}$ at $\varepsilon \approx \Delta_{g}$ is larger than in the normal case $\left(\varepsilon \gg \Delta_{g}\right.$ ), the imaginary part of $k_{T}$ has a peak. This results in a modified Hanle curve for temperatures below $T_{c}$ [see Figs. 5(c) and 5(e)], in which the suppression of the spin signal ( $R_{\text {Sy }}$ corresponding to the detector polarization $\boldsymbol{P}_{D}=P_{D} \boldsymbol{y}$ so that $\left.\boldsymbol{P}_{D} \| \boldsymbol{P}_{I} \perp \boldsymbol{h}\right)$ appears at smaller magnetic fields than in the normal state, while the oscillation becomes smaller. In contrast, if the spin-flip mechanism dominates $(\beta>0)$, the imaginary part of $k_{T}$ is suppressed at $\varepsilon \approx \Delta_{g}$ [Fig. 5(b)]. This leads to an increase of the oscillation period of $R_{\mathrm{Sy}}(h)$ when the temperature is decreased [Figs. 5(d) and 5(f)]. The real part $\operatorname{Re} k_{T}$ has a larger value than in the normal state [Fig. 5(a)]. Such an increase is mainly determined by the large renormalization of the spin relaxation in the superconducting state associated with spin-flip scattering [17,18]. In this case the spin relaxation length has a weaker dependence on the external magnetic field than in the normal state. This explains the Hanle curves shown in Figs. 5(d) and 5(f), where the decay scale of $R_{\text {Sy }}(h)$ increases towards lower temperatures at $T<T_{c}$.

At sufficiently low temperatures, the main contribution to the nonlocal resistance comes from energies below the gap in Eqs. (18) and (34), and the transport is dictated by subgap tunneling. This subgap process is different from the previously discussed process [24]. It exists only in the presence of a Zeeman field noncollinear with the injector electrode polarization. In such a system the subgap spin imbalance in the absence of a charge imbalance appears due to the gyromagnetic precession of the injected quasiparticle spins driven by the noncollinear Zeeman field. When the spin-polarized detector is connected, it converts the spin imbalance into the charge current according to Eq. (1). We neglect the corrections to the distribution functions which describe this subgap charge current in the superconducting wire. 
(a)

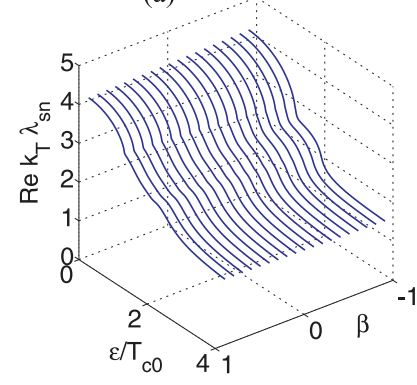

(b)
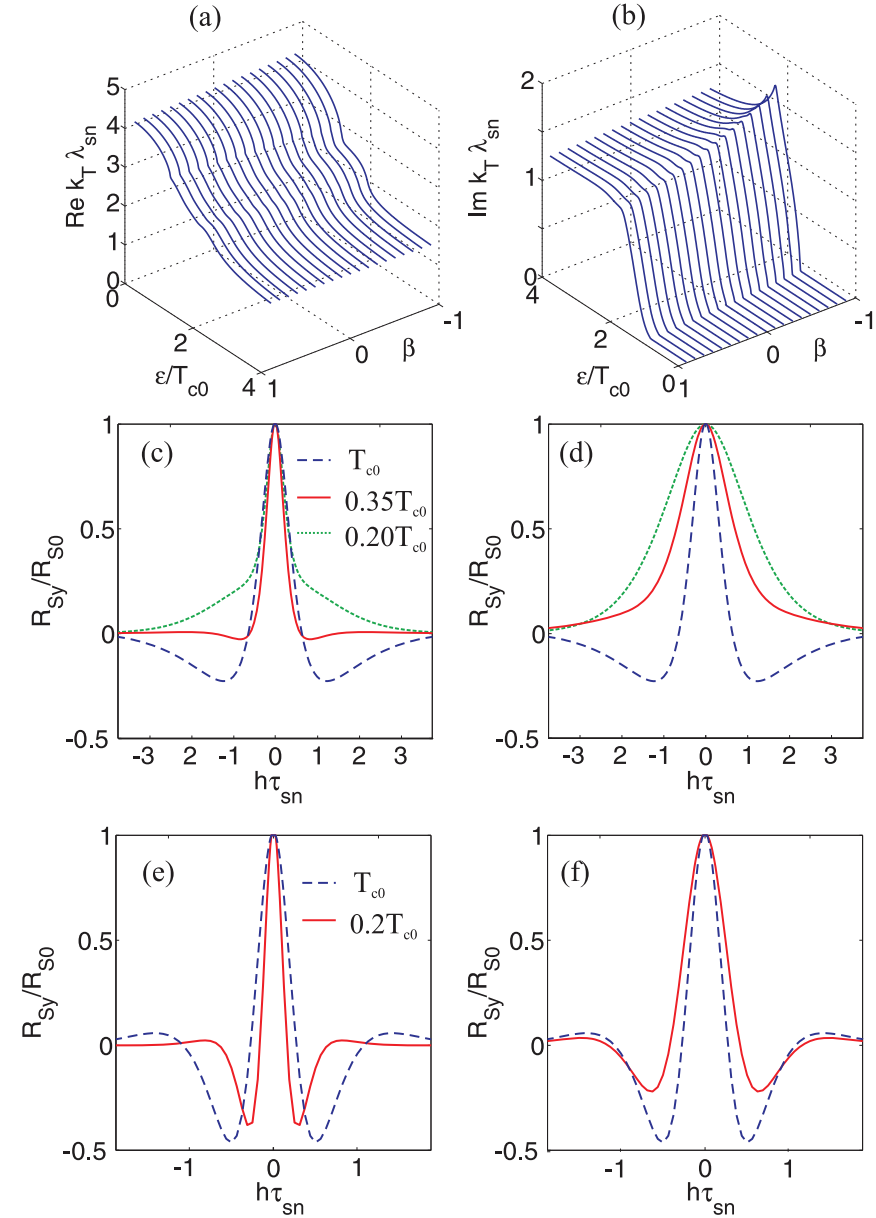

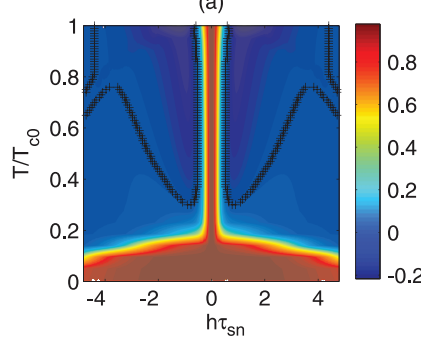

(c)

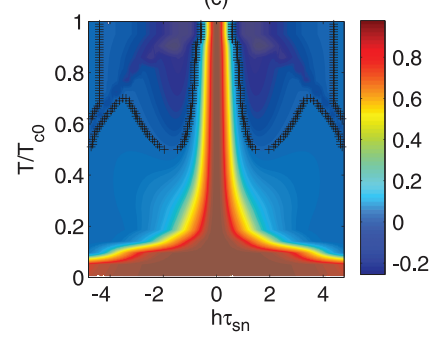

(b)

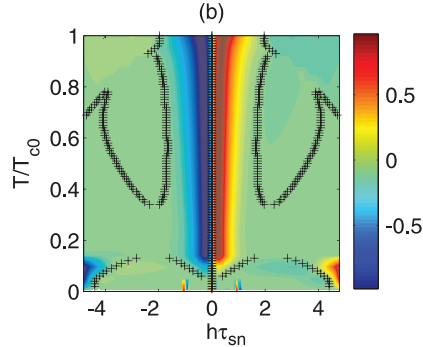

(d)

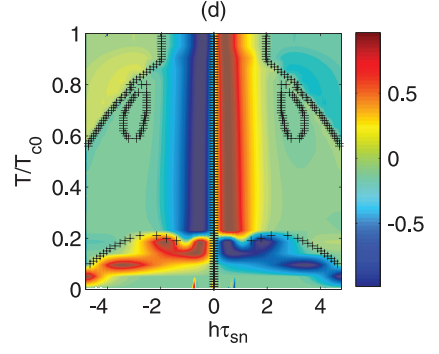

FIG. 6. (Color online) Temperature and field dependencies of the nonlocal spin signal. Panels (a) and (c) show $R_{\mathrm{Sy}}$ for $\boldsymbol{P}_{D} \| \boldsymbol{y}$, and panels (b) and (d) show $R_{\mathrm{Sx}}$ for $\boldsymbol{P}_{D} \| \boldsymbol{x}$. In (a,b) $\beta=-0.9$ and (c,d) $\beta=0.5$. The plots are normalized to the absolute maximum values at each temperature. The distance between the injector and the detector is $L_{D}=2 \lambda_{\mathrm{sn}}, \tau_{\mathrm{sn}}=5 / T_{c 0}$, and $\kappa_{I} \sqrt{D / T_{c 0}}=0.001$. The lines of zeros in $R_{\mathrm{Sx}}, R_{\mathrm{Sy}}$ are shown by black crosses.

If instead of the above-analyzed configuration one assumes that the three vectors $\left(\boldsymbol{P}_{D}, \boldsymbol{P}_{I}, \boldsymbol{h}\right)$ are perpendicular to each other (e.g., $\boldsymbol{P}_{D}=P_{D} \boldsymbol{x}, \boldsymbol{P}_{I}=P_{I} \boldsymbol{y}, \boldsymbol{h}=h \boldsymbol{z}$ ), the subgap current is absent in the detector circuit and the corresponding spin signal $R_{\mathrm{sx}}$ has a strong dependence on $h$, even in the limit $T \rightarrow 0$ [see Figs. 6(b) and 6(d)]. Note that $R_{\mathrm{Sx}}(h)=$ $-R_{\mathrm{Sx}}(-h)$ and therefore, in contrast to the usual Hanle effect, this signal can be measured without changing the magnetization of the electrodes $\boldsymbol{P}_{D}, \boldsymbol{P}_{I}$ but just by changing the sign of the external magnetic field.

\section{CONCLUSION}

In conclusion, we have developed a theoretical framework to study spin rotation and relaxation in superconductors in the case of noncollinear spin fields. We have analyzed the Hanle effect in a mesoscopic superconductor and demonstrated that the nonlocal magnetoresistance deviates from that in the normal state. Moreover, we show that the Hanle curves depend on the nature of the spin-scattering mechanism, either spin-orbit or spin-flip impurities. Our findings provide a way to identify these mechanisms by standard magnetoresistance measurements in nonlocal spin valves, and establish the fundamental physics underpinning the spin control and manipulation in superconducting devices.

\section{ACKNOWLEDGMENTS}

The work of T.T.H. was supported by the Academy of Finland and the European Research Council (Grant No. 240362Heattronics). The work of F.S.B. has been supported by the Spanish Ministry of Economy and Competitiveness under 
Project No. FIS2011-28851-C02-02. F.S.B. acknowledges funding from the Leverhulme Trust through an International
Network Grant (Grant No. IN-2013-033). P.V. acknowledges the Academy of Finland for financial support.
[1] I. Žutić, J. Fabian, and S. Das Sarma, Rev. Mod. Phys. 76, 323 (2004).

[2] D. D. Awschalom and M. E. Flatté, Nat. Phys. 3, 153 (2007).

[3] J. M. Kikkawa, D. D. Awschalom, Nature (London) 397, 139 (1999).

[4] I. Appelbaum, B. Q. Huang, and D. J. Monsma, Nature (London) 447, 295 (2007).

[5] N. Tombros, C. Jozsa, M. Popinciuc, H. T. Jonkman, and B. J. van Wees, Nature (London) 448, 571 (2007).

[6] M. Johnson and R. H. Silsbee, Phys. Rev. Lett. 55, 1790 (1985).

[7] F. J. Jedema, H. B. Heersche, A. T. Filip, J. J. A. Baselmans, and B. J. van Wees, Nature (London) 416, 713 (2002).

[8] F. J. Jedema, A. T. Filip, and B. J. van Wees, Nature (London) 410, 345 (2001).

[9] Y. Fukuma et al., Nat. Mater. 10, 527 (2011).

[10] D. H. Hernando, Yu. V. Nazarov, A. Brataas, and G. E. W. Bauer, Phys. Rev. B 62, 5700 (2000).

[11] H. Yang, S.-H. Yang, S. Takahashi, S. Maekawa, and S. S. P. Parkin, Nature Mater. 9, 586 (2010).

[12] F. Hübler, M. J. Wolf, D. Beckmann, H.v. Löhneysen, Phys. Rev. Lett. 109, 207001 (2012).

[13] C. H. L. Quay, D. Chevallier, C. Bena, and M. Aprili, Nat. Phys. 9, 84 (2013).

[14] K. Ohnishi, Y. Ono, T. Nomura, and T. Kimura, Sci. Rep. 4, 6260 (2014).

[15] M. Eschrig, Phys. Today 64, 43 (2011).

[16] N. Banerjee, C. B. Smiet, R. G. J. Smits, A. Ozaeta, F. S. Bergeret, M. G. Blamire, and J. W. A. Robinson, Nature Comm. 5, 3048 (2014).

[17] J. P. Morten, A. Brataas, and W. Belzig, Phys. Rev. B 70, 212508 (2004).
[18] J. P. Morten, A. Brataas, and W. Belzig, Phys. Rev. B 72, 014510 (2005).

[19] N. Poli, J. P. Morten, M. Urech, A. Brataas, D. B. Haviland, and V. Korenivski, Phys. Rev. Lett. 100, 136601 (2008).

[20] H. L. Zhao and S. Hershfield, Phys. Rev. B 52, 3632 (1995).

[21] T. Wakamura, N. Hasegawa, K. Ohnishi, Y. Niimi, and YoshiChika Otani, Phys. Rev. Lett. 112, 036602 (2014).

[22] G. Falci, D. Feinberg, and F. W. J. Hekking, Europhys. Lett. 54, 255 (2001).

[23] D. Beckmann, H. B. Weber, H. v. Lohneysen, Phys. Rev. Lett. 93, 197003 (2004).

[24] M. S. Kalenkov and A. D. Zaikin, Phys. Rev. B 82, 024522 (2010).

[25] S. Takahashi and S. Maekawa, Phys. Rev. B 67, 052409 (2003).

[26] The charge imbalance in Eq. (1) can be obtained from $\mu_{0}=$ $\int_{0}^{\infty} \operatorname{Tr}\left[\tau_{3}\left(g^{K}-g_{\text {eq }}^{K}\right)\right] d \varepsilon / 8$, where $g_{\text {eq }}^{K}$ is the equilibrium GF. However, it drops from all subsequent equations, as we focus on the spin accumulation.

[27] F. S. Bergeret, A. F. Volkov, and K. B. Efetov, Rev. Mod. Phys. 77, 1321 (2005).

[28] A. Schmid and G. Schön, J. Low. Temp. Phys. 20, 207 (1975).

[29] A. A. Abrikosov and L. P. Gor'kov, Zh. Eksp. Teor. Fiz. 39, 1781 (1960) [Sov. Phys. JETP 12, 1243 (1961)].

[30] D. Saint-James, D. Sarma, and E. J. Thomas, Type II Superconductivity (Pergamon, New York, 1969).

[31] F. S. Bergeret, A. Verso, and A. F. Volkov, Phys. Rev. B 86, 214516 (2012).

[32] M. Yu. Kuprianov and V. F. Lukichev, JETP 67, 1163 (1986) [Zh. Eksp.Teor. Fiz. 94, 139 (1988)].

[33] K. Maki, Phys. Rev. B 8, 191 (1973). 